\title{
The impact of emotion on prospective memory and monitoring: No pain, big gain
}

\author{
Cynthia May • Max Owens • Gilles O. Einstein
}

Published online: 26 July 2012

(C) Psychonomic Society, Inc. 2012

\begin{abstract}
The emotionally enhanced memory effect is robust across studies of retrospective memory, with heightened recall for items with emotional content (e.g., words like "murder") relative to neutral items (e.g., words like "envelope"). Only a handful of studies have examined the influence of emotion on prospective memory (PM), with mixed results. In some cases emotion enhances PM, and in others it impairs PM. Interpretation of these findings is clouded by methodological differences across studies and by the fact that, to date, no study has examined the impact of emotion on PM monitoring. In our study, we assessed PM performance when PM targets were neutral, negative, and positive, and also investigated monitoring across these different PM target types. Participants showed heightened PM performance for positive and negative relative to neutral targets, yet there was no evidence of additional monitoring for emotional targets. In fact, measures of monitoring were significantly reduced when the PM targets were emotional rather than neutral. Our findings suggest that it is possible to boost PM performance in a focal task using emotional cues, and that the use of emotional cues reduces the need for monitoring.
\end{abstract}

Keywords Prospective memory $\cdot$ Emotion $\cdot$ Human memory

C. May $(\bowtie)$

Department of Psychology, College of Charleston,

66 George Street,

Charleston, South Carolina 29424, USA

e-mail: mayc@cofc.edu

\section{Owens}

Medical University of South Carolina,

Charleston, South Carolina, USA

G. O. Einstein

Department of Psychology, Furman University,

Greenville, South Carolina, USA
Prospective memory (PM) involves remembering to perform an intention at a certain point in the future (e.g., taking medication with a meal), and PM failures can be embarrassing (e.g., forgetting a social appointment) or even life threatening (e.g., forgetting to turn off the oven). In the laboratory, PM investigations typically require that participants remember to execute a specific future intention (e.g., pressing a key when a target word appears) while simultaneously engaging in an ongoing task (e.g., lexical decision). Successful PM requires not only memory for the intention, but also the initiation of the act at the appropriate moment (e.g., Einstein, Holland, McDaniel, \& Guynn, 1992; Einstein \& McDaniel, 1996, 2005; Kliegel, McDaniel, \& Einstein, 2000). Some intentions may need to be fulfilled when a certain event takes place (event-based PM), while others must occur at a specific time (time-based PM; e.g., Einstein \& McDaniel, 2005). This study explored whether emotionally valenced cues improve event-based prospective remembering.

Researchers have recently begun to investigate whether emotion enhances cue saliency in a PM task, and consequently improves PM performance. A number of related findings have suggested that emotion should indeed boost cue saliency, as studies have shown that emotional targets are detected faster than neutral targets (e.g., Öhman, Flykt, \& Esteves, 2001; Öhman, Lundqvist, \& Esteves, 2001), that emotion can enhance visual processing (e.g., Phelps, Ling, \& Carrasco, 2006), and that emotional items are remembered better than neutral items in a retrospective memory task (e.g., Buchanan \& Adolphs, 2004; Cahill \& McGaugh, 1995; Denburg, Buchanan, Tranel, \& Adolphs, 2003; Grühn, Smith, \& Baltes, 2005; Ochsner, 2000). One would expect, then, that emotion might increase the distinctiveness or saliency of a PM target, thereby improving target detection.

A handful of studies have examined the influence of emotion on PM performance, but the findings have been 
mixed (e.g., Altgassen, Phillips, Henry, Rendell, \& Kliegel, 2010; Clark-Foos, Brewer, Marsh, Meeks, \& Cook, 2009; Rendell et al., 2011). Unfortunately, methodological differences across these studies, along with limitations in some of the paradigms, prevent a clear understanding of the role of emotion in PM, particularly with respect to the influence that emotion might have on cue saliency. For example, Clark-Foos et al. found that PM performance was better when cues were positively rather than negatively valenced. However, they did not include a neutral baseline for comparison, and thus it was impossible to ascertain whether either positive or negative cues would have produced an advantage over neutral cues.

In another experiment in their study, Clark-Foos et al. (2009) used neutral cues embedded in either neutral or emotional contexts. Here, participants read sentences (e.g., "Those who got diarrhea had eaten oysters") and were instructed to press a key whenever they encountered a food item (here, "oysters"). The results from this study showed that neutral PM cues embedded in neutral contexts were detected more often than neutral PM cues embedded in emotional contexts. Because the emotion was associated with the context (e.g., "diarrhea") rather than with the PM cue (e.g., "oysters"), it was not possible to determine the influence of emotion on cue saliency per se. Furthermore, related research has demonstrated that emotion heightens memory for central or intrinsic features of a stimulus or event but impairs memory for peripheral or extrinsic features (Christianson, 1992; Christianson, Loftus, Hoffman, \& Loftus, 1991; Heuer \& Reisberg, 1990; Kensinger, 2007; Kensinger, Garoff-Eaton, \& Schacter, 2007; Loftus, 1979). For example, people who view a picture of a snake in the forest show strong memory for the snake itself, but not for its surroundings, presumably because attention is devoted to the snake at a cost to the surrounding context (Kensinger, 2007). Thus, it is not surprising that neutral targets embedded in emotional contexts led to poorer PM, as attention was likely diverted from the neutral items to the emotional context.

Some evidence has spoken more directly to the prospect that performance is better for emotional than for neutral cues in event-based PM tasks (Altgassen et al., 2010; Rendell et al., 2011). Altgassen et al. demonstrated better PM for emotional than for neutral PM targets, although this finding was reliable for older but not younger adults. In addition, Rendell et al. found significantly better PM for positive than for neutral cues for both younger and older adults, but the difference between negative and neutral cues was not reliable. Both studies indeed suggested that emotional PM cues may be more salient than neutral cues, at least in some circumstances, but the methodologies across these studies limited any strong conclusions that could be drawn about PM and emotion. For example, only four stimuli (two positive and two negative) were used to assess the influence of emotion on performance in the Altgassen et al. study. Furthermore, in both the Altgassen and Rendell studies, the emotional PM cues were no more arousing than the neutral PM cues. As arousal plays a key role in the impact of emotion on cognitive performance (e.g., Ochsner, 2000; Reisberg, Heuer, MacLean, \& O'Shaughnessy, 1988; Thomas \& LaBar, 2005), the use of nonarousing emotional PM cues in those studies may have resulted in a smaller effect of emotion on PM. Thus, although these results were suggestive, further research is necessary before we can conclude with confidence that emotional cues benefit PM. In the present research, we used highly arousing emotional cues and sampled from among eight instances of each emotional category.

Beyond determining whether emotional cues enhance PM, another goal was to explore the cognitive processes that underlie benefits of emotional PM cues. According to the multiprocess theory (McDaniel \& Einstein, 2000, 2007), prospective memory retrieval can be accomplished through a variety of processes, ranging from capacity-consuming monitoring processes (e.g., actively monitoring for the target event while performing the ongoing task) to relatively automatic spontaneous-retrieval processes (in which processing of the target cue triggers retrieval of the intention in the absence of monitoring). It seems plausible that emotional PM cues could improve PM either by encouraging monitoring or by enhancing spontaneous-retrieval processes. For example, to the extent that emotional cues are highly salient and arousing, they could foster greater interest and focus on the PM task and increased monitoring. Previous research has shown that instructions that emphasize the importance of the PM task lead to higher levels of task interference or slowing on the ongoing task (relative to instructions that deemphasize the PM task), and researchers have interpreted this task interference as reflecting preparatory attentional processes or monitoring (Einstein et al., 2005; Loft, Kearney, \& Remington, 2008; Smith \& Bayen, 2004). From this perspective, any improved PM performance with emotional cues should be associated with increased monitoring.

On the other hand, emotional cues could benefit spontaneous-retrieval processes. Previous research has shown that salient or distinctive PM cues lead to very high PM performance (Brandimonte \& Passolunghi, 1994; Cherry et al., 2001; Cohen, Dixon, Lindsay, \& Masson, 2003; Einstein \& McDaniel, 1990; Einstein, McDaniel, Manzi, Cochran, \& Baker, 2000; McDaniel \& Einstein, 1993), and that this high performance can occur with no evidence of monitoring (Harrison \& Einstein, 2010). If emotion operates to boost the saliency of PM targets, one would expect that emotion, like other cue manipulations (e.g., perceptual distinctiveness), would reduce the demand 
for deliberate monitoring and stimulate retrieval via spontaneous-retrieval processes. If this were the case, the benefits of emotional PM cues should be accompanied by no increases in monitoring, and perhaps by decreases. These views have been difficult to evaluate in previous research because no previous work on PM and emotion has examined the consequences of emotional PM cues on monitoring.

The present study was designed to assess PM performance for neutral and highly arousing emotional targets and to evaluate whether monitoring varied with the type of PM target. Our study used a modified version of the lexical decision paradigm pioneered by Marsh, Hicks, and Watson (2002). Our participants engaged in an ongoing lexical decision task (LDT) and were instructed to press a key whenever a PM target appeared during the LDT. Participants completed four blocks of lexical decision trials, including a control block (no PM task) and three different PM blocks (neutral targets, highly arousing positive targets, and highly arousing negative targets). Monitoring was assessed by comparing LDT performance (accuracy and response times) across blocks.

\section{Method}

\section{Participants}

A group of 40 undergraduates (ages 18-25 years) from the College of Charleston participated in this experiment as one way to fulfill a course credit.

\section{Materials}

The stimuli for the LDT included 240 nonwords and 240 words. The nonwords were drawn from the English Lexicon Project (Balota et al., 2007) nonword generator, were between four and ten letters long, and were pronounceable to English speakers (e.g., "beflect"). The words were selected from the Affective Norms for English Words database (ANEW; Bradley \& Lang, 1999), which provides ratings for the arousal levels of words, on a scale of 1-9 (with 1 being least arousing and 9 being most arousing), the valence of words, on a scale of 1-9 (with 1 being most negative and 9 being most positive), and the frequency of words in standard American English writing (using the Kučera \& Francis, 1967, values). One-third of the 240 words used in this study had a neutral valence (range 4-6; $M=4.97, S D=0.51$ ), one-third had a negative valence rating (all scores $<4 ; M=2.95, S D=0.68$ ), and one-third had a positive valence rating (all scores $>6 ; M=7.03, S D=$ $0.60)$. Although the words differed in valence, all nontarget LDT items were selected to be moderate to low in arousal (all scores $<5.5$ ), and arousal levels did not differ across word valences, $F<1$. Similarly, word frequency did not vary across word valences, $F<1$.

The LDT stimuli were divided into four blocks of lexical decision trials, with each block containing 60 nonwords and 60 words (20 positive, 20 negative, and 20 neutral). The nonwords and words were each repeated once within a given block, for a total of 240 lexical decision trials per block.

Three of the four LDT blocks included two PM target words, which were also drawn from the ANEW database (Bradley \& Lang, 1999). The fourth block served as a control block, providing a baseline for the LDT task. For the PM blocks, each of the two PM target words was repeated once within the block, for a total of four PM trials per block. For blocks containing PM targets, the first target appeared after the 50th trial, and at least 50 trials intervened between each of the four PM targets.

The valences of the PM targets varied across blocks, with one block containing only neutral PM targets (e.g., "kettle" and "corridor"), one block containing only positive, highly arousing PM targets (e.g., "passion" and "miracle"), and one block containing only negative, highly arousing PM targets (e.g., "panic" and "murderer"). The two PM target words used in each block were drawn from pools of eight neutral words $(M$ : arousal $=3.6$, valence $=5.1)$, eight highly arousing positive words $(M$ : arousal $=7.5$, valence $=8.2)$, or eight highly arousing negative words $(M$ : arousal $=7.5$, valence $=$ 2.2). Word frequency $(M=19.8)$ and length $(M=6.2)$ were equated across PM target types. Valence ratings varied significantly across PM target types, $F(2,23)=303.1, p<.001$. The negative and positive PM targets did not vary in arousal $(F<1)$, but both were significantly more arousing than the neutral targets (both $p \mathrm{~s}<.001$ ).

Within each block, the PM target words replaced lexical decision words of the same valence, so that all blocks consisted of 240 trials with equal proportions of positive, negative, and neutral words. The PM targets were counterbalanced across participants so that the eight PM targets within each valence were used an equal number of times across the study. We used multiple PM target items to ensure that our results did not reflect idiosyncratic properties of specific targets.

We used partial counterbalancing to vary the order of block type (control, positive PM, negative PM, or neutral PM) across participants, so that each block type was presented equally often as the first, second, third, or fourth block of trials across participants, thus controlling for both practice and fatigue effects in LDT responding.

To introduce a delay between the PM instructions and the onset of the LDT trials, we presented paper-and-pencil questionnaires as filler tasks. These tasks were presented at the beginning of each block and included a vocabulary test, a health and education questionnaire, and a creativity task 
that required participants to create designs for different objects (flags, flowerpots, etc.).

\section{Procedure}

All of the participants completed the LDT and the PM task via computer, and E-Prime (Version 2; Psychology Software Tools, Pittsburgh, PA) was used to implement the experiment. Participants first completed a consent form and then read the instructions for the LDT. The participants were informed that they were to make word/nonword judgments about strings of letters that would appear individually on the computer screen. Participants were told to press the key with the green sticker (here, the " $J$ " key) if the string was a word, and to press the key with the red sticker (here, the "F" key) if the string was not a word. Speed of response on the LDT was emphasized as being the primary task for this study. The participants completed 10 practice trials and were then given instructions for the first block of critical trials.

If the participants' first block was a control block, they were reminded of the LDT instructions and of the emphasis on speeded responding once more, and were then given a 4min filler task. If the participants' first block was a PM block, they were instructed that in addition to the LDT task, they had the secondary task of pressing the key with the yellow sticker (here, the " $T$ " key) whenever they saw one of two target words (e.g., "miracle" or "passion"). The participants then repeated back these instructions, along with the target words designated for that block, and then were given a 4-min filler task. After the filler task, all participants completed the block of LDT trials. No further mention was made of the PM task. At the completion of each block, participants repeated this sequence for the next block of trials, with PM instructions (two new targets per block) followed by a 4-min filler task and then the LDT and PM tasks.

\section{Results}

\section{PM performance}

PM responses were counted as correct if they were initiated during viewing of the PM cue or within three trials after the
PM cue appeared. Late responses (those occurring after the PM cue was already off screen) made up less than $1 \%$ of the responses, and there were no significant differences in late responding across the valences of PM cues. Removing late responses from other results did not alter the pattern of the findings in any way. Mean PM performance for the different kinds of cues is reported in Table 1. A one-way repeated measures analysis of variance (ANOVA) indicated a main effect of PM cue condition, $F(2,78)=5.98, p<.004, \eta^{2}=$ .13. Further analyses showed that PM performance was reliably higher with positive than with neutral cues, $F(1$, $39)=8.7, p<.005, \eta^{2}=.18$, and similarly that PM was significantly better with negative than with neutral cues, $F$ $(1,39)=6.8, p<.01, \eta^{2}=.15$. PM did not differ across the positive and negative cues, $F<1$.

\section{Ongoing-task performance}

Accuracy and response times in the LDT were used to assess monitoring, and the mean scores on these measures are reported in Table 1. As can be seen in the table, accuracy rates were generally high in all conditions; nonetheless, a one-way repeated measures ANOVA indicated a main effect of cue condition, $F(3,117)=7.8, p<.001, \eta^{2}=.16$. Accuracy rates were higher for the control block than for the neutral PM block, $F(1,39)=15.9, p<.001, \eta^{2}=.29$, for the positive PM block, $F(1,39)=14.8, p<.001, \eta^{2}=.28$, and for the negative PM block, $F(1,39)=15.4, p<.001, \eta^{2}=.28$. No other differences in accuracy rates were significant, $F_{\mathrm{s}}<1$. Thus, accuracy rates were better in the control block than in all of the PM blocks, but did not differ across the different PM conditions.

Preparatory attentional processing or monitoring costs are typically reflected in the speed of performing the ongoing task (Marsh, Hicks, Cook, Hansen, \& Pallos, 2003; Smith \& Bayen, 2004). Response times were evaluated only for LDT trials with correct responses on word trials, and the response times were trimmed to exclude responses that were $2.5 S D$ s beyond the mean for each participant. Furthermore, trials that included a PM target, or on which a participant made a PM response, were also not included in the response time analyses. A repeated measures ANOVA indicated a main effect of block condition on response times, $F(3$, $117)=21.4, p<.001, \eta^{2}=.35$. Further analyses showed

Table 1 Mean proportions of prospective memory (PM) responses made by PM cue type, with accuracy percentages and response times (RTs; standard deviations in parentheses) for the lexical decision task

\begin{tabular}{lllll}
\hline PM Cue Type & Control & Neutral & Positive & Negative \\
\hline Proportion of PM responses & N/A & $28.8(27.4)$ & $50.0(33.0)$ & $44.4(28.6)$ \\
LDT accuracy & $93.4(0.03)$ & $91.4(0.04)$ & $91.3(0.04)$ & $92.0(0.04)$ \\
LDT RT (in ms) & $789.9(136.3)$ & $900.6(163.2)$ & $868.1(133.5)$ & $868.8(138.9)$ \\
\hline
\end{tabular}


that response times were significantly faster in the control block than in any of the PM blocks: neutral, $F(1,39)=70.5$, $p<.001, \eta^{2}=.64$; positive, $F(1,39)=34.7, p<.001, \eta^{2}=$ .47 ; or negative, $F(1,39)=35.8, p<.001, \eta^{2}=.48$. In addition, response times for the positive PM block were significantly faster than those for the neutral PM block, $F$ $(1,39)=6.4, p=.015, \eta^{2}=.14$. Similarly, response times in the negative PM block were marginally faster than those in the neutral PM block, $F(1,39)=3.0, p<.09, \eta^{2}=.09$. Response times did not differ between the positive and negative PM blocks, $F<1$.

\section{Discussion}

For the present study, we assessed event-based PM performance when PM targets were neutral, positive, and negative, and also evaluated monitoring across these different PM target types. Participants engaged in an ongoing lexical decision task as their primary task, and in addition they performed a PM task in three of the four LDT blocks. The nature of the PM cues varied across these three blocks, with each block containing either neutral cues, high-arousing positive cues, or high-arousing negative cues. The results indicated reliably greater PM success for positive and negative cues than for neutral cues. These findings are consistent with data from Altgassen et al. (2010) and Rendell et al. (2011) in showing that PM can be enhanced when targets are emotional rather than neutral in nature.

We note that Altgassen et al. (2010) demonstrated this enhancement only for older adults, whereas Rendell et al. (2011) demonstrated it for both younger and older adults, but for positive items only. It is possible that differences in their methodologies contributed to these limited effects. As noted earlier, Altgassen et al. included only four emotional stimuli (two positive and two negative), and these emotional stimuli were all nonarousing. Similarly, the emotional stimuli used in the Rendell et al. study were also nonarousing. Here, we used 16 emotional stimuli, all of which were highly arousing according to the ANEW norms (Bradley \& Lang, 1999). As arousal is known to influence stimulus memorability (e.g., Denberg et al., 2003; Grühn et al., 2005), and studies demonstrating the influence of emotion on attention have tended to use very arousing stimuli (e.g., Öhman, Flykt, \& Esteves, 2001; Phelps et al., 2006), we believe that the arousing nature of our emotional stimuli may be responsible for the more robust effects observed here.

Our findings are consistent with a large literature suggesting that emotional information enjoys a relatively privileged status in a number of different cognitive processes (e.g., Buchanan \& Adolphs, 2004; Cahill \& McGaugh, 1995; Dimberg \& Öhman, 1996; Hamann, 2001; Öhman,
Lundqvist, \& Esteves, 2001; Phelps et al., 2006), and they extend this privileged status to PM as well. The data indicate that emotion may be another factor that boosts cue saliency, thus reducing the need for deliberate monitoring and simultaneously boosting PM success (e.g., Brandimonte \& Passolunghi, 1994; McDaniel \& Einstein, 1993; West, Wymbs, Jakubek, \& Herndon, 2003). We emphasize that our manipulation of emotion was specific to PM cues, and not to the emotional state of the participants. Indeed, a very different pattern might emerge when the emotional state of participants is varied, as people in a happy mood tend to show diminished PM success, while those in a sad mood tend to show elevated PM success (Rummel, Hepp, Klein, \& Silberleitner, 2012).

The pattern of monitoring findings also suggests that emotion boosts cue saliency. Whereas monitoring was evident for all PM blocks, the increase in PM success observed for emotional targets was not associated with increased monitoring. Instead, monitoring was reduced for emotional relative to neutral targets, suggesting that the elevated PM for emotional cues was not a consequence of heightened vigilance. Rather, the data are consistent with the notion that emotional items are more distinctive or salient than neutral items, and that they may spontaneously trigger the PM intention and reduce the need for deliberate processing. Beyond demonstrating that emotional cues lead to more efficient PM than do neutral cues, the significantly lower monitoring levels with emotional cues suggest that participants have some metacognitive awareness of their saliency.

PM performance involves both retrospective and prospective components, and thus it is possible that heightened retrospective memory for the PM targets contributed to greater PM success. Indeed, emotional items are often recalled better than neutral items in retrospective tasks (e.g., Cahill \& McGaugh, 1995; Grühn et al., 2005; Ochsner, 2000). We note, however, that participants had only two PM targets to remember per block, that they repeated the $\mathrm{PM}$ instructions and the target words, and that other PM research has suggested that retrospective memory for two-item target lists with neutral items is very high, often at ceiling (Breneiser \& McDaniel, 2006; Cohen, Jaudas, \& Gollwitzer, 2008; Rummel, Einstein, \& Rampey, in press). Thus, while heightened retrospective memory could have contributed to the enhanced PM performance observed here, it is unlikely to be the sole source of the benefit.

Whereas previous research suggested that participants often do not monitor when their PM task includes one or two target events (Cohen et al., 2008), our results demonstrated significant levels of monitoring in all three PM conditions. This may have been due to our use of a withinsubjects design (Cohen et al., 2008, used a between-subjects design), wherein participants performed three PM blocks and each block had a new set of cues. In order to keep the 
right cues in mind for each block, the participants probably had to rehearse the cues occasionally. Despite the fact that reliable monitoring did occur in this study, our findings of higher PM with emotional cues, along with reduced monitoring for those cues, indicate that the performance benefit was not due to enhanced monitoring.

Our emotional cues were both emotionally valenced and arousing, and at present, it is unclear whether the emotional valence, the arousing value, or both were responsible for improving PM. It is also unclear at this point which of the possible spontaneous-retrieval mechanisms proposed by McDaniel and Einstein $(2000,2007)$ were responsible for the improvement in PM with positive and negative cues. A plausible interpretation is that an emotional and arousing cue captures attention (e.g., Öhman, Flykt, \& Esteves, 2001; Öhman, Lundqvist, \& Esteves, 2001; Phelps et al., 2006), much like salient or distinctive cues in other PM studies (e.g., Brandimonte \& Passolunghi, 1994; Einstein \& McDaniel, 1990; McDaniel \& Einstein, 1993), thus heightening PM success. Regardless of the ultimate interpretation, however, it is clear that emotional and arousing cues substantially improve PM while also decreasing the need for capacity-consuming monitoring processes.

Emotional PM cues thus may be particularly beneficial in circumstances in which monitoring is difficult to maintain (e.g., in the midst of a busy workday) or requires a change in a well-practiced routine or skill. Consider the parent who, instead of taking the usual route to work, must change routine and bring an infant child to daycare first. As one's commute gets underway and work responsibilities fill conscious thought, there is a risk of forgetting about the child, especially if the child falls asleep. To ensure execution of this important task, the parent may want to place an emotional cue (e.g., a picture of the child, or even a dirty diaper) in the front of the car. Future investigations may want to explore the utility of PM cues in more applied settings, particularly for PM tasks in which failure comes at a high cost (e.g., medication adherence or financial deadlines).

\section{References}

Altgassen, M., Phillips, L. H., Henry, J. D., Rendell, P. G., \& Kliegel, M. (2010). Emotional target cues eliminate age differences in prospective memory. Quarterly Journal of Experimental Psychology, 63, 1057-1064.

Balota, D. A., Yap, M. J., Cortese, M. J., Hutchison, K. A., Kessler, B., Loftis, B., \& Treiman, R. (2007). The English Lexicon Project. Behavior Research Methods, 39, 445-459. doi:10.3758/ BF03193014

Bradley, M. M., \& Lang, P. J. (1999). Affective norms for English words (ANEW): Stimuli, instruction manual and affective ratings (Technical Report No. C-1). Gainesville, FL: University of Florida, NIMH Center for Research in Psychophysiology.
Brandimonte, M. A., \& Passolunghi, M. C. (1994). The effect of cuefamiliarity, cue-distinctiveness, and retention interval on prospective remembering. Quarterly Journal of Experimental Psychology, 47A, 565-587. doi:10.1080/14640749408401128

Breneiser, J. E., \& McDaniel, M. A. (2006). Discrepancy processes in prospective memory retrieval. Psychonomic Bulletin \& Review, 13, 837-841. doi:10.3758/BF03194006

Buchanan, T. W., \& Adolphs, R. (2004). The neuroanatomy of emotional memory in humans. In D. Reisberg \& P. Hertel (Eds.), Memory and emotion (pp. 42-75). New York, NY: Oxford University Press.

Cahill, L., \& McGaugh, J. L. (1995). A novel demonstration of enhanced memory associated with emotional arousal. Consciousness and Cognition, 4, 410-421.

Cherry, K. E., Martin, R. C., Simmons-D'Gerolamo, S. S., Pinkston, J. B., Griffing, A., \& Gouvier, W. D. (2001). Prospective remembering in younger and older adults: Role of the prospective cue. Memory, 9, 177-193.

Christianson, S.-Å. (1992). Emotional stress and eyewitness memory: A critical review. Psychological Bulletin, 112, 284-309.

Christianson, S.-Å., Loftus, E. F., Hoffman, H., \& Loftus, G. R. (1991). Eye fixations and memory for emotional events. Journal of Experimental Psychology: Learning, Memory, and Cognition, 17, 693-701. doi:10.1037/0278-7393.17.4.693

Clark-Foos, A., Brewer, G. A., Marsh, R. L., Meeks, J. T., \& Cook, G. I. (2009). The valence of event-based prospective memory cues or the context in which they occur affects their detection. American Journal of Psychology, 122, 89-97.

Cohen, A.-L., Dixon, R. A., Lindsay, D. S., \& Masson, M. E. (2003). The effect of perceptual distinctiveness on the prospective and retrospective components of prospective memory in young and old adults. Canadian Journal of Experimental Psychology, 57, 274-289.

Cohen, A.-L., Jaudas, A., \& Gollwitzer, P. M. (2008). Number of cues influences the cost of remembering to remember. Memory \& Cognition, 36, 149-156. doi:10.3758/MC.36.1.149

Denburg, N. L., Buchanan, T. W., Tranel, D., \& Adolphs, R. (2003). Evidence for preserved emotional memory in normal older persons. Emotion, 3, 239-253.

Dimberg, U., \& Öhman, A. (1996). Behold the wrath: Psychophysiological responses to facial stimuli. Motivation and Emotion, 20, $149-182$.

Einstein, G. O., Holland, L. J., McDaniel, M. A., \& Guynn, M. J. (1992). Age-related deficits in prospective memory: the influence of task complexity. Psychology and Aging, 7, 471-478.

Einstein, G. O., \& McDaniel, M. A. (1990). Normal aging and prospective memory. Journal of Experimental Psychology: Learning, Memory, and Cognition, 16, 717-726.

Einstein, G. O., \& McDaniel, M. A. (1996). Retrieval processes in prospective memory: Theoretical approaches and some new empirical findings. In M. Brandimonte, G. O. Einstein, \& M. A. McDaniel (Eds.), Prospective memory: Theory and applications (pp. 115-142). Mahwah, NJ: Erlbaum.

Einstein, G. O., \& McDaniel, M. A. (2005). Prospective memory: Multiple retrieval processes. Current Directions in Psychological Science, 14, 286-290. doi:10.1111/j.0963-7214.2005.00382.x

Einstein, G. O., McDaniel, M. A., Manzi, M., Cochran, B., \& Baker, M. (2000). Prospective memory and aging: Forgetting intentions over short delays. Psychology and Aging, 15, 671-683. doi:10.1037/0882-7974.15.4.671

Einstein, G. O., McDaniel, M. A., Thomas, R., Mayfield, S., Shank, H., Morrisette, N., \& Breneiser, J. (2005). Multiple processes in prospective memory retrieval: Factors determining monitoring versus spontaneous retrieval. Journal of Experimental Psychology: General, 134, 327-342. doi:10.1037/0096-3445.134.3.327

Grühn, D., Smith, J., \& Baltes, P. B. (2005). No aging bias favoring memory for positive material: Evidence from a heterogeneity- 
homogeneity list paradigm using emotionally toned words. Psychology and Aging, 20, 579-588. doi:10.1037/08827974.20.4.579

Hamann, S. (2001). Cognitive and neural mechanisms of emotional memory. Trends in Cognitive Sciences, 5, 394-400.

Harrison, T. L., \& Einstein, G. O. (2010). Prospective memory: Are preparatory attentional processes necessary for a single focal cue? Memory \& Cognition, 38, 860-867. doi:10.3758/MC.38.7.860

Heuer, F., \& Reisberg, D. (1990). Vivid memories of emotional events: The accuracy of remembered minutiae. Memory \& Cognition, 18, 496-506.

Kensinger, E. A. (2007). Negative emotion enhances memory accuracy: Behavioral and neuroimaging evidence. Current Directions in Psychological Science, 16, 213-218. doi:10.1111/j.1467-8721.2007.00506.x

Kensinger, E. A., Garoff-Eaton, R. J., \& Schacter, D. L. (2007). How negative emotion enhances the visual specificity of a memory. Journal of Cognitive Neuroscience, 19, 1872-1887.

Kliegel, M., McDaniel, M. A., \& Einstein, G. O. (2000). Plan formation, retention, and execution in prospective memory: A new approach and age-related effects. Memory \& Cognition, 28, 1041-1049.

Kučera, H., \& Francis, W. N. (1967). Computational analysis of present day American English. Providence, RI: Brown University Press.

Loft, S., Kearney, R., \& Remington, R. (2008). Is task interference in event-based prospective memory dependent on cue presentation? Memory \& Cognition, 36, 139-148. doi:10.3758/MC.36.1.139

Loftus, E. F. (1979). The malleability of human memory. American Scientist, 67, 312-320.

Marsh, R. L., Hicks, J. L., Cook, G. I., Hansen, J. S., \& Pallos, A. L. (2003). Interference to ongoing activities covaries with characteristics of an event-based intention. Journal of Experimental Psychology: Learning, Memory, and Cognition, 29, 861-870. doi:10.1037/02787393.29.5.861

Marsh, R. L., Hicks, J. L., \& Watson, V. (2002). The dynamics of intention retrieval and coordination of action in event-based prospective memory. Journal of Experimental Psychology. Learning, Memory, and Cognition, 28, 652-659.

McDaniel, M. A., \& Einstein, G. O. (1993). The importance of cue familiarity and cue distinctiveness in prospective memory. Memory, 1, 23-41.

McDaniel, M. A., \& Einstein, G. O. (2000). Strategic and automatic processes in prospective memory retrieval: A multiprocess framework. Applied Cognitive Psychology, 14, S127-S144. doi:10.1002/acp.775

McDaniel, M. A., \& Einstein, G. O. (2007). Prospective memory: An overview and synthesis of an emerging field. Thousand Oaks, CA: Sage Publications.

Ochsner, K. N. (2000). Are affective events richly recollected or simply familiar? The experience and process of recognizing feelings past. Journal of Experimental Psychology: General, 129, 242-261. doi:10.1037/0096-3445.129.2.242

Öhman, A., Flykt, A., \& Esteves, F. (2001a). Emotion drives attention: Detecting the snake in the grass. Journal of Experimental Psychology: General, 130, 466-478. doi:10.1037/0096-3445.130.3.466

Öhman, A., Lundqvist, D., \& Esteves, F. (2001b). The face in the crowd revisited: A threat advantage with schematic stimuli. Journal of Personality and Social Psychology, 80, 381-396.

Phelps, E. A., Ling, S., \& Carrasco, M. (2006). Emotion facilitates perception and potentiates the perceptual benefits of attention. Psychological Science, 17, 292-299.

Reisberg, D., Heuer, F., MacLean, J., \& O'Shaughnessy, M. (1988). The quantity, not the quality, of affect predicts memory vividness. Bulletin of the Psychonomic Society, 26, 100-103.

Rendell, P. G., Phillips, L. H., Henry, J. D., Brumby-Rendell, T., Garcia, X. P., Altgassen, M., \& Kliegel, M. (2011). Prospective memory, emotional valence and ageing. Cognition and Emotion, 25, 916-925.

Rummel, J., Einstein, G. O., \& Rampey, H. (in press). Implementation intention encoding in a prospective memory task enhances spontaneous retrieval of intentions. Memory.

Rummel, J., Hepp, J., Klein, S. A., \& Silberleitner, N. (2012). Affective state and event-based prospective memory. Cognition \& Emotion, 26, 351-361.

Smith, R. E., \& Bayen, U. J. (2004). A multinomial model of eventbased prospective memory. Journal of Experimental Psychology: Learning, Memory, and Cognition, 30, 756-777. doi:10.1037/ 0278-7393.30.4.756

Thomas, L. A., \& LaBar, K. S. (2005). Emotional arousal enhances word repetition priming. Cognition \& Emotion, 19, 1027-1047. doi:10.1080/02699930500172440

West, R., Wymbs, N., Jakubek, K., \& Herndon, R. W. (2003). Effects of intention load and background context on prospective remembering: An event-related brain potential study. Psychophysiology, 40, 260-276. 